\title{
Deterministically entangling distant nitrogen-vacancy centers by a nanomechanical cantilever
}

\author{
Z. Y. $\mathrm{Xu}^{1,2}$, Y. M. Hu${ }^{1,2}$, W. L. Yang ${ }^{1,2}$, M. Feng ${ }^{1}$ 田 and J. F. Du田 \\ ${ }^{1}$ State Key Laboratory of Magnetic Resonance and Atomic and Molecular Physics, \\ Wuhan Institute of Physics and Mathematics, Chinese Academy of Sciences, Wuhan 430071, China \\ ${ }^{2}$ Graduate School of the Chinese Academy of Sciences, Beijing 100049, China and \\ ${ }^{3}$ Hefei National Laboratory for Physics Sciences at Microscale and Department of Modern Physics, \\ University of Science and Technology of China, Hefei, 230026, China
}

\begin{abstract}
We present a practical scheme by global addressing to deterministically entangle negatively charged nitrogen-vacancy $(\mathrm{N}-\mathrm{V})$ centers in distant diamonds using a nano-mechanical cantilever with the magnetic tips strongly coupled to the N-V electron spins. Symmetric Dicke states are generated as an example, and the experimental feasibility and challenge of our scheme are discussed.
\end{abstract}

PACS numbers: 03.67.Bg, 03.67.Lx, 71.55.-i, 76.30.Mi

Over past years, negatively charged nitrogen-vacancy $(\mathrm{N}-\mathrm{V})$ centers in diamond have been considered as one of the most promising candidates for solid-state quantum information processing (QIP) due to their long coherence time at room temperature [1]. The intensive experimental studies have been paid on quantum logical gating [2], state storage and transfer [3], and preparation of entangled states [4], using the electron-spin and the nearby nuclear spins within a single diamond.

In the context of a large-scale diamond-based QIP, the generation of multipartite entangled states among distant N-V centers is of particular importance and experimental challenge. One of the proposed approaches is to use the measurement-based QIP scheme [5]. By detecting the emitted photons which have been entangled with different $\mathrm{N}-\mathrm{V}$ electron spins, distant $\mathrm{N}-\mathrm{V}$ centers can be entangled in a nondeterministic way. Such an approach has the major advantage that no direct interactions among qubits are required, which implies a relatively easy control. An alternative is the scalable optical coupling between $\mathrm{N}-\mathrm{V}$ centers and high-Q microcavities [6]. By sharing the common modes of a microcavity or sharing flying single-photon pulses, the remote N-V centers could be coherently manipulated or entangled, which may open up the possibilities of N-V-based scalable QIP.

To have an efficient processing of quantum information, however, we prefer deterministic manipulations. In the present work, we will show the possibility to entangle multiple $\mathrm{N}-\mathrm{V}$ centers by a nano-mechanical cantilever in a deterministic fashion. We had noticed a recent work for a strong magnetic coupling between electron-spin degrees of freedom of a $\mathrm{N}-\mathrm{V}$ center and a nano-mechanical resonator [7, 8]. In our case, we have a line of N-V centers, each of which is located in a nanoscale diamond. We will show how to generate the symmetric Dicke states [9], denoted by $\left|D_{n}^{(m)}\right\rangle$ with equally weighted superposition

\footnotetext{
*Electronic address: mangfeng@wipm.ac.cn
}

${ }^{\dagger}$ Electronic address: djf@ustc.edu.cn

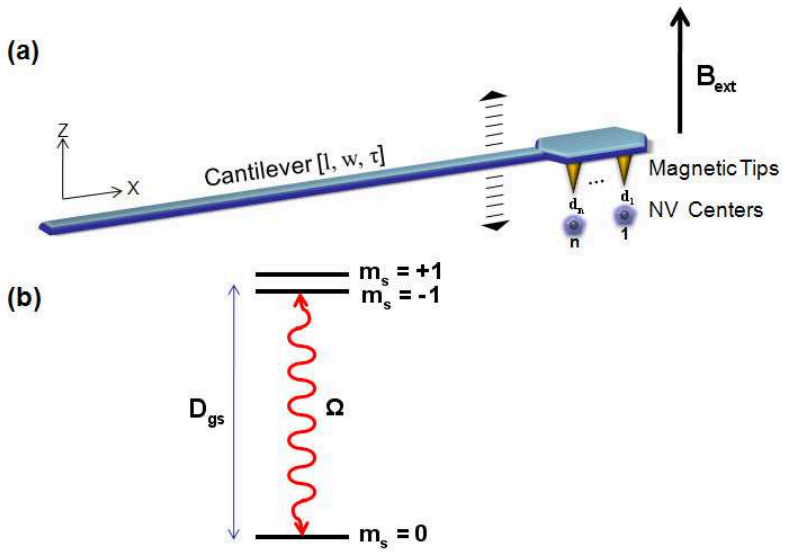

FIG. 1: (Color online) (a) Schematic of the physical system consisting of an array of $\mathrm{N}-\mathrm{V}$ centers strongly coupled to the quantized motion of a cantilever with length $l$, width $w$ and thickness $\tau$. An external magnetic field $\mathbf{B}_{\text {ext }}$ is applied along the symmetry axis of the N-V centers. (b) The energy level diagram for the ground triplet state of the electron spin within a $\mathrm{N}-\mathrm{V}$ center, with a zero-field splitting $\mathrm{D}_{g s}$ between spin levels $m_{s}=0$ and $m_{s}= \pm 1$. We restrict our study to the down state $m_{s}=0$ and the up state $m_{s}=-1$ by driving a microwave field.

of all permutations of $m$ excitations among $n$ particles, by global addressing. Such multipartite entangled states own great properties of robustness against decoherence and particle loss and have been observed experimentally with linear optics systems [10]. The favorable features of our scheme include the deterministic preparation of entangled electron-spin states between $\mathrm{N}-\mathrm{V}$ centers and the potential to be scaled up with near future technologies. Besides, as a scheme with global addressing, only a microwave field is required during the entanglement preparation, which greatly reduces the experimental time and difficulty. Furthermore, our proposed scheme could be realized with very high fidelity due to our fast manipulations and the long decoherence time of electron spins in $\mathrm{N}-\mathrm{V}$ centers. 
As sketched in Fig. 1(a), an array of evenly spaced magnetic tips are attached at the end of a nanomechanical cantilever, under which are $n$ one-to-one correspondent $\mathrm{N}-\mathrm{V}$ centers. In order to entangle the electron spins in spatially separate N-V centers by a cantilever, we have to couple the electron spins to the motion of the resonator. This can be achieved by exposing the electron spins to the magnetic field gradient created by the magnetic tips. For clarity of our description, we label $\lambda_{j}=\chi_{e} B_{g}^{j} z_{0}^{j}(j=1,2, \cdots n)$ as the coupling strength, where $B_{g}^{j}=\left|\partial_{z} \mathbf{B}_{t i p_{j}}\right|, z_{0}^{j}$ is the zero-point amplitude of the cantilever regarding the $j$ th tip and $\chi_{e}$ is the chargemass ratio of an electron. For simplicity, we assume that all the $\mathrm{N}-\mathrm{V}$ centers are equally coupled to the cantilever $\left(\lambda_{j}=\lambda\right)$, which could be achieved by elaborately adjusting the magnetic field gradient of the tips and the distances between the tips and the corresponding $\mathrm{N}-\mathrm{V}$ centers. Furthermore, in most part of the paper, we will neglect the influence from the nuclear spins, which implies a pure $\mathrm{N}-\mathrm{V}$ center without ${ }^{13} \mathrm{C}$ doped or with well polarized nuclear spins of ${ }^{13} C$ or ${ }^{15} N$ [11]. For our purpose, we encode qubits in two sublevels: $m_{s}=0$ and $m_{s}=-1$ of the ground triplet state, and a microwave field will be employed to drive the two sublevels. As a result, the Hamiltonian of the whole system can be written in units of $\hbar=1$ as

$$
\begin{aligned}
H= & \sum_{j=1}^{n} \omega_{0}|-1\rangle_{j}\langle-1|+\nu a^{\dagger} a \\
& +\sum_{j=1}^{n} \frac{\Omega_{j}(t)}{2}\left(|0\rangle_{j}\left\langle-1\left|e^{i \omega_{j} t}+\right|-1\right\rangle_{j}\langle 0| e^{-i \omega_{j} t}\right) \\
& +\sum_{j=1}^{n} \frac{\lambda}{2}\left(a+a^{\dagger}\right) \sigma_{z}^{j},
\end{aligned}
$$

where $\omega_{0} \approx D_{g s}-\chi_{e}\left|\mathbf{B}_{e x t}\right|$ is the energy splitting between sublevels $|0\rangle$ and $|-1\rangle$ under the externally applied magnetic field $\mathbf{B}_{e x t}$ along $\mathrm{z}$ axis, i.e., the quantization axis, $D_{g s}$ (around $2.87 \mathrm{GHz}$ [12]) is the zero-field splitting between the spin levels $m_{s}=0$ and $m_{s}= \pm 1$ shown in Fig. 1(b). $\nu$ and $a\left(a^{\dagger}\right)$ are the frequency and annihilation (creation) operator of the motional mode of the cantilever. $\Omega_{j}(t)$ and $\omega_{j}$ are the Rabi frequency and the frequency of the microwave regarding the cantilever coupling to the $j$ th N-V center, and $\sigma_{z}^{j}=|-1\rangle_{j}\langle-1|-$ $|0\rangle_{j}\langle 0|$. For simplicity, we may assume in following treatment $\Omega_{j}(t)=\Omega(t)$ and $\omega_{j}=\omega(j=1,2, \cdots n)$. So the Hamiltonian of the total system in the interaction picture is

$$
\begin{aligned}
H_{I}= & \sum_{j=1}^{n} \frac{\Omega(t)}{2}\left[|0\rangle_{j}\langle-1| e^{i\left(\omega-\omega_{0}\right) t}\right. \\
& \left.+|-1\rangle_{j}\langle 0| e^{-i\left(\omega-\omega_{0}\right) t}\right] \\
& +\sum_{j=1}^{n} \frac{\lambda}{2}\left(a e^{-i \nu t}+a^{\dagger} e^{i \nu t}\right) \sigma_{z}^{j}
\end{aligned}
$$

If $\omega=\omega_{0}$, Eq. (2) in a new basis $| \pm\rangle=(|-1\rangle \pm|0\rangle) / \sqrt{2}$ reads as $\sum_{j=1}^{n} \frac{\Omega(t)}{2} \tilde{\sigma}_{z}^{j}+\sum_{j=1}^{n} \frac{\lambda}{2}\left(a e^{-i \nu t}+a^{\dagger} e^{i \nu t}\right) \tilde{\sigma}_{x}^{j}$, where $\tilde{\sigma}_{x}^{j}=|+\rangle_{j}\langle-|+|-\rangle_{j}\langle+|$ and $\tilde{\sigma}_{z}^{j}=|+\rangle_{j}\langle+|-|-\rangle_{j}\langle-|$. Considering $\Omega(t)$ is comparable to $\nu$, we rewrite Eq. (2) in a rotating frame under rotating wave approximation, obtaining

$$
\tilde{H}_{I}=\sum_{j=1}^{n} \frac{\lambda}{2}\left\{a|+\rangle_{j}\langle-| \exp \left[-i \int_{t_{i}}^{t} \delta(t) d t\right]+\text { H.c. }\right\},
$$

with $\delta(t)=\nu-\Omega(t)$.

From the viewpoint of QIP, the vibrational mode of a cantilever could be considered as a common data bus for distant N-V centers. Therefore, using Eq. (3), we may achieve various kinds of multiple entangled states. As an example, we will show below how to prepare symmetric Dicke states of the electron spins in distant N-V centers by global microwave addressing.

We consider a Si cantilever beam of length $l=5 \mu \mathrm{m}$, width $w=50 \mathrm{~nm}$ and thickness $\tau=50 \mathrm{~nm}$ with effective mass $M \simeq 7.28 \times 10^{-18} \mathrm{~kg}$, resonant frequency of the lowest-order flexural mode $\nu \simeq 14.87 \mathrm{MHz}$ and the zeropoint amplitude of fluctuation $\sqrt{1 /(2 M \nu)} \simeq 6.98 \times 10^{-13}$ $\mathrm{m}$ [13]. The $\mathrm{N}$-V-cantilever coupling strength $\lambda \simeq 0.96$ $\mathrm{MHz}$ has been achieved under a magnetic field gradient $7.8 \times 10^{6} \mathrm{Tm}^{-1}$ at a distance $\simeq 25 \mathrm{~nm}$ away from the tip [7, 14]. In addition, the distance between the nearestneighbor tips is set to be around $150 \mathrm{~nm}$.

We will generate two nontrivial threeparticle symmetric Dicke states: $\left|D_{3}^{(1)}\right\rangle=$ $(|+--\rangle+|-+-\rangle+|--+\rangle) / \sqrt{3}$ and $\left|D_{3}^{(2)}\right\rangle=$ $(|++-\rangle+|+-+\rangle+|-++\rangle) / \sqrt{3}$. We get started from Eq. (3), which can be rewritten as $\tilde{H}_{I}=$ $\frac{\lambda}{2} \sum_{j=1}^{3}\left(a|+\rangle_{j}\left\langle-\left|+a^{\dagger}\right|-\right\rangle_{j}\langle+|\right)-\delta(t) \sum_{j=1}^{3}|+\rangle_{j}\langle+|$ after performing a time-dependent phase transformation.

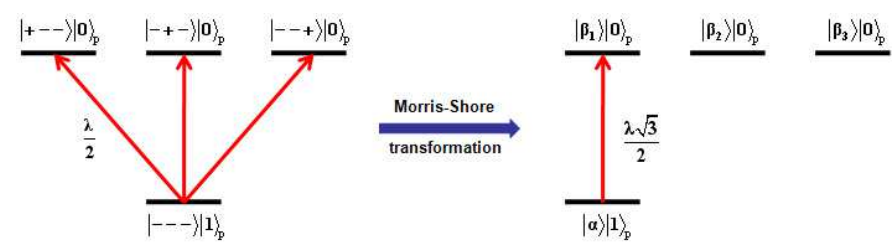

FIG. 2: (Color online) Left: The energy level pattern of a string of three NV centers with an initial state $|---\rangle|1\rangle_{p}$ globally driven by a microwave field, where we set $\delta(t)=0$ and |\rangle$_{p}$ is for the motional state of the cantilever. Right: The MS transformation produces a two-level subsystem where $\left|\beta_{1}\right\rangle=\left|D_{3}^{(1)}\right\rangle,|\alpha\rangle=|---\rangle$ and two decoupled dark states $\left|\beta_{2}\right\rangle=(|+--\rangle-2|-+-\rangle+|--+\rangle) / \sqrt{6}$ and $\left|\beta_{3}\right\rangle=(\mid+$ $-\rangle-|--+\rangle) / \sqrt{2}$.

Let us first consider how to prepare $\left|D_{3}^{(1)}\right\rangle$. We assume that the vibrational state of the cantilever has been cooled to the Fock state $|1\rangle_{p}[, 7,8]$ and the electron-spin states of $\mathrm{N}-\mathrm{V}$ centers are initially prepared in $|---\rangle$. By means of Morris-Shore (MS) transformation [15], it is possible to factorize the Hamiltonian into a smaller closed subspace [16]. As shown in Fig. 2, in MS basis the original system is decomposed to a two-level subsystem plus two independent single-level subsystems. The effective 
Hamiltonian of the two-level subsystem is written as

$$
H_{\text {eff }}^{(1)}=\left(\begin{array}{cc}
-\delta(t) & \frac{\sqrt{3}}{2} \lambda \\
\frac{\sqrt{3}}{2} \lambda & 0
\end{array}\right)
$$

in the basis $\left\{\left|\beta_{1}\right\rangle|0\rangle_{p},|\alpha\rangle|1\rangle_{p}\right\}$, where $\left|\beta_{1}\right\rangle=\left|D_{3}^{(1)}\right\rangle$ and $|\alpha\rangle=|---\rangle$. The top state $\left|\beta_{1}\right\rangle$ is connected to the initial state $|\alpha\rangle$ with an effective coupling strength $\sqrt{3} \lambda / 2$ and can be interchanged by a microwave field addressing the three $\mathrm{N}-\mathrm{V}$ centers globally. When $\delta(t)$ is changed to zero (by using a nearly square pulse of the microwave), the desired $\left|D_{3}^{(1)}\right\rangle$ can be realized at $\mathrm{t}=\pi /(\sqrt{3} \lambda)$ with unit probability.

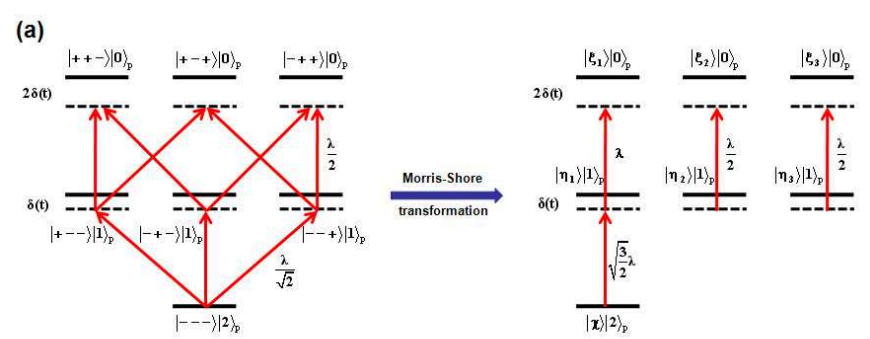

(b)

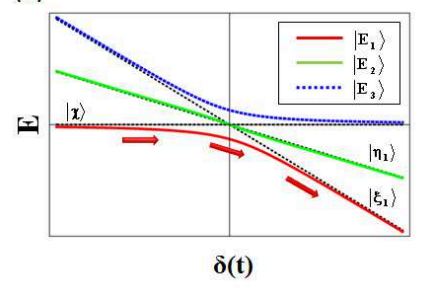

(c)

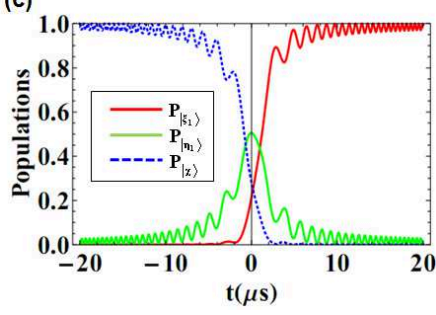

FIG. 3: (Color online) (a) Left: The energy level pattern of a string of three NV centers with an initial state $|---\rangle|2\rangle_{p}$ globally driven by a microwave field. Right: The MS transformation produces a three-level subsystem where $\left|\xi_{1}\right\rangle=\left|D_{3}^{(2)}\right\rangle$, $\left|\eta_{1}\right\rangle=\left|D_{3}^{(1)}\right\rangle,|\chi\rangle=|---\rangle$ and two decoupled two-level subsystems $\left|\xi_{2}\right\rangle=(|++-\rangle-2|+-+\rangle+|-++\rangle) / \sqrt{6}$, $\left|\eta_{2}\right\rangle=(|+--\rangle-2|-+-\rangle+|--+\rangle) / \sqrt{6}$ and $\left|\xi_{3}\right\rangle=$ $(|++-\rangle-|-++\rangle) / \sqrt{2},\left|\eta_{3}\right\rangle=(|+--\rangle-|--+\rangle) / \sqrt{2}$. (b) Adiabatic evolution of the three-level subsystem, where the three black-dotted lines represent $\left|\xi_{1}\right\rangle,\left|\eta_{1}\right\rangle$ and $|\chi\rangle$ respectively. The up state $\left|\xi_{1}\right\rangle$ and ground state $|\chi\rangle$ can be interchanged by adiabatic passages (arrows) under the adiabatic condition. (c) Populations of states $\left|\xi_{1}\right\rangle,\left|\eta_{1}\right\rangle,|\chi\rangle$ during the adiabatic evolution.

Now we turn to the preparation of $\left|D_{3}^{(2)}\right\rangle$. In contrast to $\left|D_{3}^{(1)}\right\rangle$ preparation, the state of the whole system in this case is initially prepared in $|---\rangle|2\rangle_{p}$. After performing MS transformation, we obtain a three-level subsystem plus two additional two-level subsystems [See Fig. 3(a)]. The effective Hamiltonian reads as

$$
H_{\text {eff }}^{(2)}=\left(\begin{array}{ccc}
-2 \delta(t) & \lambda & 0 \\
\lambda & -\delta(t) & \lambda \sqrt{\frac{3}{2}} \\
0 & \lambda \sqrt{\frac{3}{2}} & 0
\end{array}\right)
$$

in the basis $\left\{\left|\xi_{1}\right\rangle|0\rangle_{p},\left|\eta_{1}\right\rangle|1\rangle_{p},|\chi\rangle|2\rangle_{p}\right\}$, where $\left|\xi_{1}\right\rangle=$ $\left|D_{3}^{(2)}\right\rangle,\left|\eta_{1}\right\rangle=\left|D_{3}^{(1)}\right\rangle$ and $|\chi\rangle=|---\rangle$. It is easy to check by setting $\delta(t)=0$ that the population probability of $\left|D_{3}^{(2)}\right\rangle$ will only reach 0.95 . In order to generate $\left|D_{3}^{(2)}\right\rangle$ with higher probability, we will employ adiabatic techniques as below. The top state $\left|\xi_{1}\right\rangle$ and the bottom state $|\chi\rangle$ in Fig. 3(b) can be interchanged through adiabatic passages under the adiabatic condition: $\max _{t \in\left[t_{i}, t_{f}\right]}\left|\left\langle E_{l}(t)\left|\partial_{t} H_{\text {eff }}^{(2)}\right| E_{k}(t)\right\rangle /\left[E_{k}(t)-E_{l}(t)\right]^{2}\right| \ll 1$ $(l \neq k)$, where $\left|E_{k}\right\rangle$ and $E_{k}$ are the instantaneous eigenstates and eigeN-Values of Eq. (5). In Fig. 3(c), the populations of $\left|\xi_{1}\right\rangle,\left|\eta_{1}\right\rangle$ and $|\chi\rangle$ are numerically calculated from Eq. (5), where we have assumed a purely linear microwave chirp (using sawtooth pulse of the microwave). By addressing the three $\mathrm{N}-\mathrm{V}$ centers globally, we could achieve the population probability of $\left|D_{3}^{(2)}\right\rangle$ to be higher than 0.99 through this adiabatic passage.

With similar steps to the above cases for three N-V centers, a generalization to $\left|D_{n}^{(m)}\right\rangle$ with $n>m>3$ is straightforward.

In the above treatment, we have assumed the $\mathrm{N}-\mathrm{V}$ centers to be equally coupled to the cantilever. In realistic case, however, the coupling should be slightly different due to imprecision. Figure 4(a) demonstrates a simplified case for the fidelities of the produced states under the relative error of the coupling strength $\Delta$, defined by $\lambda_{n}=[1-(n-1) \Delta] \lambda$, where the fidelities still remain high in the case of the small relative error.

The main decoherence sources associated with our implementation are dephasing of the electron spins and the intrinsic heating of the cantilever. Since our operation for the symmetric Dicke states could be accomplished on the time scale of $1.8 \sim 30 \mu \mathrm{s}$, which is much shorter than the reported electron-spin relaxation time $T_{1}=6 \mathrm{~ms}$ [4] as well as the dephasing time $T_{2}=350 \mu \mathrm{s}$ [17] induced by the nuclear-spin fluctuation inside a $\mathrm{N}-\mathrm{V}$ center. Thus, the influence on the $\mathrm{N}-\mathrm{V}$ electron-spin from the intrinsic damping and dephasing is negligible. On the other hand, as the cantilever is ultra-sensitive to the magnetic force generated between the tips and the N-V electron spins [13, 14, 18], we will concentrate on the heating effect of the cantilever by the master equation $\partial_{t} \rho=-i\left[H_{\text {eff }}^{(k)}, \rho\right]+\mathrm{L} \rho$ with $k=1,2$ [corresponding to Eqs. (4) and (5), respectively], where $\mathrm{€} \rho=\Gamma\left(\bar{n}_{t h}+1\right)\left(2 a \rho a^{\dagger}-a^{\dagger} a \rho-\rho a^{\dagger} a\right) / 2+$ $\Gamma \bar{n}_{t h}\left(2 a^{\dagger} \rho a-a a^{\dagger} \rho-\rho a a^{\dagger}\right) / 2, \bar{n}_{t h}=1 /\left[\exp \left(\frac{\hbar \nu}{k_{B} T}\right)-1\right]$ and $k_{B}$ is the Boltzmann constant. In general, the heating factor of the cantilever is described by $\Gamma=\nu / Q$ [13], where $\nu$ and $Q$ are the vibrational frequency and the quality factor of a cantilever. Numerical calculations are performed for the fidelities of the Dicke states in Fig. 4(b), where the fidelities of $\left|D_{3}^{(1)}\right\rangle$ and $\left|D_{3}^{(2)}\right\rangle$ could reach 0.998 and 0.996 respectively in the case of the $Q$ value $10^{5}$. If the $Q$ value is lowered to $10^{4}$, the fidelity drops to 0.985 for $\left|D_{3}^{(1)}\right\rangle$ and to 0.957 for $\left|D_{3}^{(2)}\right\rangle$. 
(a)

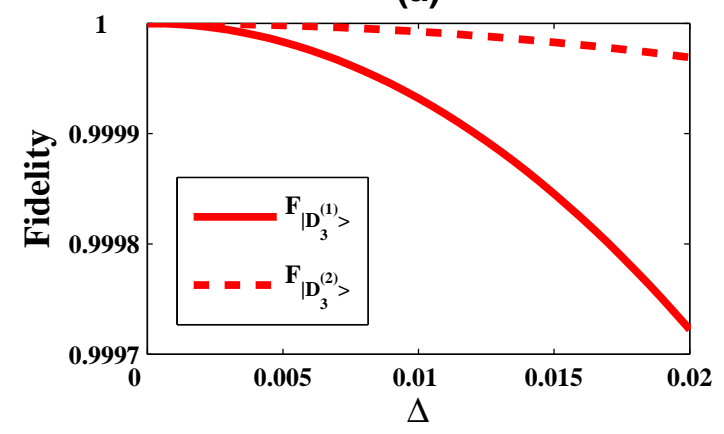

(b)

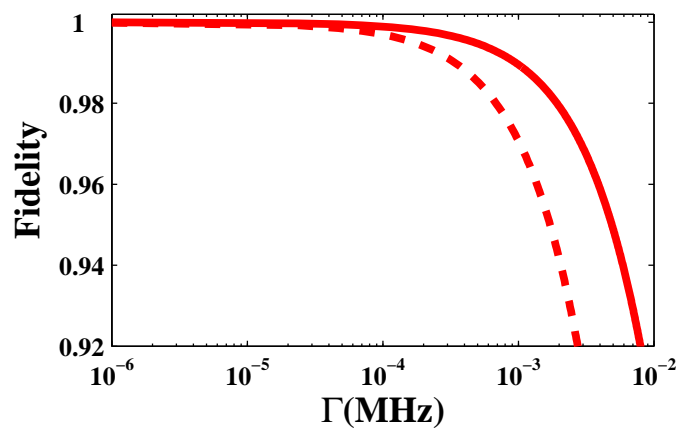

FIG. 4: (Color online) The fidelity of symmetric Dicke states $\left|D_{3}^{(1)}\right\rangle$ (solid line) and $\left|D_{3}^{(2)}\right\rangle$ (dashed line) versus (a) relative error $\Delta$ of the coupling strength; (b) heating factor $\Gamma$ of the cantilever.

We address some remarks for experimental issues of our scheme. To make sure that the N-V centers are all strongly and nearly equally coupled to the cantilever, we have to restrict the tips within a small region near the head of the cantilever, which implies a limitation of the number for available $\mathrm{N}-\mathrm{V}$ centers (at most tens of $\mathrm{N}-\mathrm{V}$ centers in our present design). So a potential for scalability with our scheme might be to combine with the non-deterministic method [5]. Besides, we require nanoscale diamonds with a single $\mathrm{N}-\mathrm{V}$ center in each and the N-V centers should be located very close to the surface of the diamonds. To our knowledge, such diamond nanoparticles are not in principle unavailable although they have not yet been achieved experimentally. On the other hand, due to the small spacing between adjacent $\mathrm{N}-\mathrm{V}$ centers, individual addressing with a microwave field is of difficulty. As a result, although entanglement could be achieved with global operations, to carry out universal quantum computing, we may have to employ the nearfield optical technique [19] with spatial resolution of the order of tens of nanometers, which had been widely used in optics and surface science, and recently also employed for QIP [20]. Alternatively, a magnetic field gradient along $\mathrm{X}$-axis would help to distinguish different qubits in frequency space. Thus universal QIP is available, and robust quantum gating, such as composite pulse method 21] or bang-bang control 22], employed previously could be straightforwardly applied to our scheme. Finally, cooling a cantilever with many magnetic tips to a required low temperature (114 $\mu K$ in our design) could be of great technical difficulty with current technology but may be resolved by recently presented cooling schemes [7, 8] .

Further consideration would iN-Volve nuclear spins which are good at storing quantum information due to the long decoherence time [23]. In this sense, we may also consider to encode the qubits in the nuclear spins, and employ the electron spins as ancillas. The hyperfine interaction helps to transfer the generated entanglement of electron spins to the corresponding nuclear spins of $\mathrm{N}-\mathrm{V}$ centers [3].

To conclude, we have proposed a scheme to deterministically entangle a string of $\mathrm{N}-\mathrm{V}$ centers by strongly coupling to the quantized motion of a nano-mechanical cantilever. Due to the long relaxation and dephasing time of the electron spins in $\mathrm{N}-\mathrm{V}$ centers together with the weak intrinsic heating effects of the cantilever, our present scheme would enable a promising way for future $\mathrm{N}-\mathrm{V}$-center-based QIP.

The work is supported by NNSFC under Grant No. 10774042 , by CAS, and by NFRPC.
[1] F. Jelezko et al., Phys. Rev. Lett. 92, 076401 (2004); R. Hanson et al., Phys. Rev. Lett. 97, 087601 (2006); L. Childress et al., Science 314, 281 (2006); M. Stoneham, Physics 2, 34 (2009).

[2] F. Jelezko et al., Phys. Rev. Lett. 93, 130501 (2004).

[3] M. V. Gurudev Dutt et al., Science 316, 1312 (2007).

[4] P. Neumann et al., Science 320, 1326 (2008).

[5] S. C. Benjamin et al., Laser \& Photon. Rev. (published online, DOI: 10.1002/lpor.200810051).

[6] Y.-S. Park et al., Nano Lett. 6, 2075 (2006); C. F. Wang et al., Appl. Phys. Lett. 90, 081110 (2007); S. Schietinger et al., Nano Lett. 8, 3911 (2008); A. Young et al., New
J. Phys. 11, 013007 (2009).

[7] P. Rabl et al., Phys. Rev. B 79, 041302(R) (2009).

[8] J. Wrachtrup, Nat. Phys. 5, 248 (2009).

[9] R. H. Dicke, Phys. Rev. 93, 99 (1954).

[10] N. Kiesel et al., Phys. Rev. Lett. 98, 063604 (2007); R. Prevedel et al., Phys. Rev. Lett. 103, 020503 (2009); W. Wieczorek et al., Phys. Rev. Lett. 103, 020504 (2009).

[11] V. Jacques et al., Phys. Rev. Lett. 102, 057403 (2009).

[12] N. B. Manson et al., Phys. Rev. B 74, 104303 (2006).

[13] J. A. Sidles et al., Rev. Mod. Phys. 67 , 249 (1995).

[14] H. J. Mamin et al., Nat. Nanotechnol. 2 , 301 (2007).

[15] J. R. Morris et al., Phys. Rev. A 27, 906 (1983); A. A. 
Rangelov et al., Phys. Rev. A 74, 053402 (2006).

[16] A. Retzker et al., Phys. Rev. A 75, 022312 (2007); I. E. Linington et al., Phys. Rev. A 77, 010302(R) (2008).

[17] T. Gaebel et al., Nat. Phys. 2, 408 (2006).

[18] B. C. Stipe et al., Phys. Rev. Lett. 86 , 2874 (2001).

[19] G. Kaupp, Atomic Force Microscopy, Scanning Near field Optical Microscopy and Nanoscratching: Application to Rough and Natural Surfaces (Springer, Heidel- berg, 2006).

[20] M. Feng et al., Europhys. Lett. 66, 14 (2004).

[21] J. A. Jones, Phil. Trans. R. Soc. Lond. A 361, 1429 (2003).

[22] L. Viola et al., Phys. Rev. A 58, 2733 (1998).

[23] N. A. Gershenfeld et al., Science 275, 350 (1997); B. E. Kane, Nature (London) 393, 133 (1998). 